\title{
DISCUSSÕES SOBRE ANÁLISE ANALÍTICA DA TRAJETÓRIA DE EQUILÍBRIO DE ESTRUTURAS DE BARRA SIMPLES SUJEITAS A GRANDES DESLOCAMENTOS: UMA ABORDAGEM ENERGÉTICA
}

\section{DISCUSSIONS ON ANALYTICAL ANALYSIS OF THE EQUILIBRIUM PATH OF SIMPLE BAR STRUCTURES UNDER TO LARGE DISPLACEMENTS: AN ENERGETIC APPROACH}

Recebido em: 01/11/2018.

Aceito em: 23/11/2018.
Túlio Raunyr Cândido Felipe ${ }^{1}$ Kaliel Gomes Andrande ${ }^{2}$ Rodolfo Baculle Januário ${ }^{3}$ Marcos Antônio Simões ${ }^{4}$

\section{RESUMO}

O objetivo deste trabalho é avaliar e debater sobre a trajetória de equilíbrio não linear geométrico de estruturas formadas por elementos de barras simples via método da energia mecânica. Para tal, os potenciais de energias são escritos em função das posições nodais dos elementos. Ademais, é empregado uma descrição Lagrangeana Total para deduzir a energia de deformação em termos das posições nodais. Consequentemente, são avaliados dois problemas estáticos sem dissipação de energia para validar a abordagem proposta. Por fim, conclui-se que em regime de deslocamentos moderados a grandes deve-se realizar uma análise não linear geométrica para mensurar a estabilidade da estrutura com relação aos pontos limites que apareceram na sua trajetória de equilíbrio.

Palavras-chave: Descrição Lagrangeana Total. Método da energia mecânica. Não linearidade geométrica.

1 Doutorando em Engenharia de Estruturas pela Escola de Engenharia de São Carlos, Universidade de São Paulo (USP). Docente da Universidade Brasil.

E-mail: tulio-raunyr@usp.br

2 Graduando em Engenharia Civil pelo Centro Universitário de João Pessoa (UNIPÊ).

E-mail: kaliel.gomes@hotmail.com

3 Graduando em Engenharia Civil pela Universidade Brasil.

E-mail: rodolfo.baccule@gmail.com

4 Graduando em Engenharia Civil pela Universidade Brasil.

E-mail: marcosimoes01@hotmail.com 


\section{ABSTRACT}

The goal of this work is to evaluate and discuss the geometric nonlinear equilibrium path of structures formed by simple bar elements by way the mechanical energy method. For this, the potentials of energies are written according to the nodal positions of the elements. Also, a Total Lagrangian description is used to measure the strain energy regarding the nodal positions. Thus, two static problems without energy dissipation are evaluated to validate the proposed approach. Finally, concluded that in a moderate to large displacement regime, a nonlinear geometric analysis must be performed to measure the stability of the structure about the limit points that appeared in its equilibrium path.

Keywords: Total Lagrangian description. mechanical energy method. geometric nonlinearity.

\section{INTRODUÇÃO}

Nos problemas de engenharia as hipóteses assumidas para representar os problemas físicos nem sempre produzem uma resposta exata do fenômeno estudado. Isso porque na mecânica do continuo as equações diferenciais parciais que descrevem tais fenômenos nem sempre tem solução analítica fechada. Dessa forma, utilizam-se métodos numéricos para aproximar tais soluções. Todavia, os métodos numéricos são soluções aproximadas das equações diferenciais, equações essas que podem já ter sofrido alguma simplificação. Assim sendo, existem várias simplificações no modelo.

Tradicionalmente a análise estrutural é realizada considerando as condições de equilíbrio na configuração inicial do corpo (posição indeformada), isto é, assumindo linearidade geométrica. Entretanto, em situações em que se tenha estruturas esbeltas e/ ou para grandes deslocamentos, tal pressuposição não é atendida. Desse modo, para uma análise robusta da trajetória de equilíbrio, o corpo deve ser analisado na configuração atual (posição deformada). A avaliação das condições de equilíbrio na configuração atual é denominada de análise não linear geométrica (CODA, 2018; PROENÇA, 2016; CRISFIELD, 1991). Consequentemente, tal análise leva em conta a trajetória exata do equilíbrio do corpo (BONET \& WOOD, 2008).

\section{OBJETIVOS}

O objetivo deste trabalho é analisar e discutir sobre a trajetória de equilíbrio não linear geométrica de estruturas formadas por elementos de barras simples via método da energia mecânica total. 


\section{METODOLOGIA}

Inicialmente são deduzido os potenciais de energia em termos das posições nodais dos elementos de barra simples em uma formulação Lagrangeana Total. Em seguida, as condições de equilíbrio são obtidas pelo princípio da estacionariedade da energia mecânica. Por fïm, para validar a presente metodologia, são empregados dois problemas acadêmicos, nos quais os resultados são discutidos em todas as suas características.

\section{ENERGIA MECÂNICA TOTAL}

O problema não linear geométrico pode ser formulado a partir da energia mecânica total contida em um corpo. Essa energia é definida pelas parcelas da energia cinética $(K)$, energia de deformação $(U)$, energia de dissipação $(\Psi)$ e potencial das forças externas (P). A primeira parcela está associada ao movimento do corpo. A segunda parcela está relacionada com a energia interna armazenada no corpo. A terceira parcela está ligada ao processo de dissipação de energia que ocorre em sistemas não conservativos. Por fim, a quarta parcela está concatenada às forças externas que são aplicadas ao corpo. Dessa maneira, a energia mecânica é dada pela seguinte expressão:

$$
\Pi=P+U+K+\Psi
$$

Para problemas conservativos, isto é, onde não há dissipação, a Equação (1) resulta:

$$
\Pi=P+U+K
$$

Neste trabalho são analisados problemas estáticos sem dissipação, portanto a Equação (2) é escrita da seguinte maneira:

$$
\Pi=P+U
$$

Pelo princípio da estacionariedade, o qual assegura que a variação do potencial de energia mecânica é nula, obtêm-se as equações de equilíbrio, isto é:

$$
\frac{\partial \Pi}{\partial \vec{Y}} \cdot \delta \vec{Y}=\frac{\partial P}{\partial \vec{Y}} \cdot \delta \vec{Y}+\frac{\partial U}{\partial \vec{Y}} \cdot \delta \vec{Y}=\left(\frac{\partial P}{\partial \vec{Y}}+\frac{\partial U}{\partial \vec{Y}}\right) \cdot \delta \vec{Y}=\overrightarrow{0}
$$

sendo $\mathbf{Y}$ o vetor das posições atuais do corpo. Como $\delta \boldsymbol{Y}$ é arbitrário, resulta:

$$
\frac{\partial \Pi}{\partial \vec{Y}}=\frac{\partial P}{\partial \vec{Y}}+\frac{\partial U}{\partial \vec{Y}}=-\vec{F}^{e x t}+\vec{F}^{\text {int }}=\overrightarrow{0}
$$


Da Equação (5), define-se a força interna $\mathbf{F}^{\text {int }}$ como a derivada da energia de deformação com relação à posição, enquanto a força externa $\mathbf{F}^{\text {ext }}$ como a derivada do potencial das forças externas em referência a posição.

A segunda derivada da Equação (5), para forças externas conservativas, é dada por:

$$
\mathbf{H}=\frac{\partial \Pi}{\partial \vec{Y} \otimes \partial \vec{Y}}=\frac{\partial U}{\partial \vec{Y} \otimes \partial \vec{Y}}
$$

em que H, simétrica, é a matriz Hessiana (CODA, 2018). Verifica-se que o equilíbrio é estável se, e somente se, no ponto de equilíbrio, $\mathbf{H}$ for positiva definida. Logo, conclui-se que o equilíbrio de uma estrutura é estável quando a configuração atual dessa estrutura constitui um mínimo local para o potencial de energia mecânica total.

O potencial de forças externas concentradas em uma formulação posicional é definido por:

$$
P=-\vec{F}^{e x t} \cdot \vec{Y}
$$

A energia de deformação em uma formulação Lagrangeana Total em função das posições nodais do corpo, é escrita da seguinte maneira:

$$
U(\vec{Y})=\int_{V_{0}} u_{e}(\vec{Y}) d V_{0}
$$

em que $u_{e}$ é a energia específica de deformação para materiais elásticos, enquanto $V_{o}$ é o volume inicial do corpo. Se existe explicitamente uma expressão para $u_{e}$, o material é dito hiperelástico (CODA, 2018).

Para a Lei de Hooke uniaxial, a expressão da energia específica de deformação resulta:

$$
u_{e}=\frac{\sigma_{0} \varepsilon}{2}=\frac{E \varepsilon^{2}}{2}
$$

onde $\sigma_{0}$ é a tensão longitudinal de engenharia; $\varepsilon$ é a deformação longitudinal de engenharia e $E$ é o modulo de elasticidade longitudinal. Consequentemente, a tensão conjugada energética de $\varepsilon$ é dada por:

$$
\sigma_{0}=\frac{d u_{e}}{d \varepsilon}=E \varepsilon
$$

Da Equação (6), depreende-se que a Lei de Hooke pode ser escrita por um potencial quadrático, também denominado de convexo. Na teoria da elasticidade linear, os potenciais assumidos como geradores de leis constitutivas consistentes devem ser convexos (CODA, 2018; OGDEN, 1984). 
Define-se a deformação longitudinal uniaxial de engenharia por:

$$
\varepsilon=\frac{d y-d x}{d x}=\frac{d y}{d x}-1=\lambda-1
$$

em que $d x$ é o comprimento inicial de um infinitésimo do corpo descarregado; $d y$ é o comprimento atual desse infinitésimo para o corpo solicitado, enquanto $\lambda$ é definido como o estiramento de Cauchy-Green, no qual é a medida fundamental de deformação (OGDEN, 1984). Verifica-se que se $\lambda>1$, tem-se tração. Por outro lado se $\lambda<1$, temse compressão no corpo. Torna-se evidente que para problemas com sentido físico, o estiramento de Cauchy-Green deve ser maior que zero $(\lambda>0)$, uma vez que o corpo não pode degenerar. Ademais, como o comprimento de referência é o inicial, aquela medida de deformação é classificada como Lagrangeana.

Combinando as Equações (5) e (6), obtém-se:

$$
U=\int_{V_{0}} \frac{E \varepsilon^{2}}{2} d V_{0}=\frac{E \varepsilon^{2}}{2} \cdot V_{0}=\frac{E \varepsilon^{2}}{2} \cdot l_{0} \cdot A_{0}
$$

sendo $l_{o}$ e $A_{o}$, respectivamente, o comprimento inicial e a área inicial do elemento de barra simples.

\section{EXEMPLOS DE APLICAÇÕES}

\section{VIGA RÍGIDA EM ENGASTE ELÁSTICO}

A Figura 1-a) apresenta uma viga rígida vinculada por engaste elástico com comprimento $l_{0}$. Após aplicação da força externa $F^{\text {ext }}$, a barra assumi uma nova configuração no espaço, conforme ilustrado na Figura 1-b). A Figura 1-c) mostra o comportamento da mola de giro, no qual essa fornece um momento fletor resistente proporcional e em sentido oposto ao giro $\alpha$ (em radianos) a que é sujeita. 
Figura 1 - a) viga rígida em engaste elástico na configuração inicial; b) viga rígida em engaste elástico na configuração atual; c) mola de giro comportamento elastico linear.

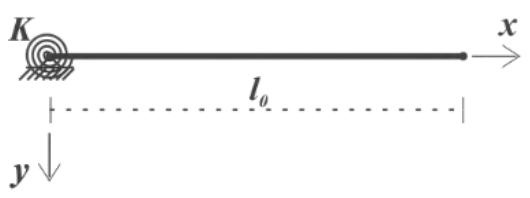

a)

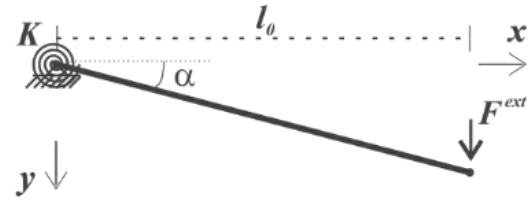

b)

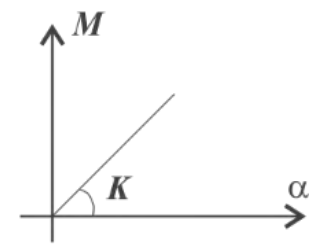

Fonte: Autores.

A solução linear deste problema é obtida via equilíbrio da viga na configuração inicial, o que resulta:

$$
\alpha=\frac{l_{0}}{K} \cdot F^{e x t}
$$

sendo $K$ a constante de mola.

A solução exata é dada pelo equilíbrio dessa viga na configuração atual, o que resulta:

$$
\frac{\alpha}{\cos (\alpha)}=\frac{l_{0}}{K} \cdot F^{e x t}
$$

A Equação (14) é não linear. Para sua solução pode-se aplicar o procedimento de Newton.

A energia mecânica para esse problema, assumindo o problema estático sem dissipação de energia, é descrita por:

$$
\Pi=P+U=-F^{e x t} \cdot l_{0} \cdot \sin (\alpha)+\frac{K \cdot \alpha^{2}}{2}
$$

ou

$$
\Pi=P+U=-F^{e x t} \cdot Y+\frac{K \cdot\left[\arcsin \left(\frac{Y}{l_{0}}\right)\right]^{2}}{2} .
$$


Pelo princípio da estacionariedade, tem-se:

$$
\left\{\begin{array}{l}
\frac{\partial \Pi}{\partial \alpha}=-F^{e x t} \cdot l_{0} \cdot \cos (\alpha)+K \cdot \alpha=0 \\
\frac{\partial \Pi}{\partial Y}=-F^{e x t}+\frac{K \cdot \arcsin \left(\frac{Y}{l_{0}}\right)}{l_{0} \cdot \sqrt{1-\left(\frac{Y}{l_{0}}\right)^{2}}}=0
\end{array}\right.
$$

Ambas as Equações (17) e (18) retornam para Equação (14). Na Equação (15) o funcional de energia foi escrito em termos do ângulo $\alpha$, logo, procede-se à derivada em função dessa incógnita, conforme a Equação (17). Por outro lado, na Equação (16), o funcional de energia foi escrito com referência as posições atuais da viga, portanto, decorre a derivada no tocante a essa incógnita, de acordo com a Equação (18).

Na Figura 2 apresenta a trajetória de equilíbrio para essa viga, assumindo $l_{0}=$ $3 \mathrm{~m}$ e $K=1000 \mathrm{KN} . \mathrm{m}$.

Figura 2 - Trajetória de equilíbrio da viga rígida com apoio elástico.

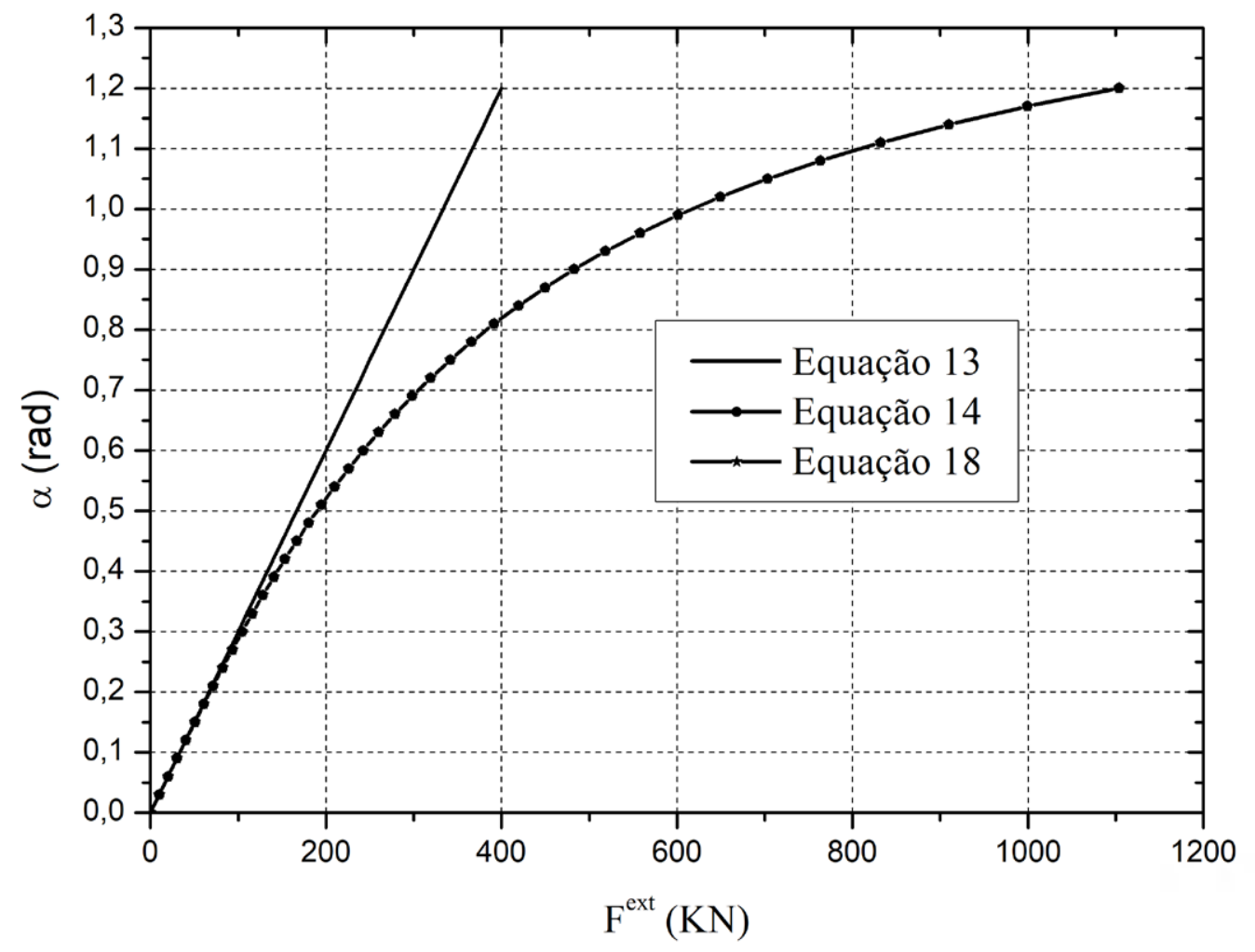

Fonte: Autores.

Note que a solução obtida pela abordagem energética (Equação 18) conduz à solução exata do problema (Equação 14), em concordância com a Figura 2. Observa-se que, para níveis de rotações pequenos, a solução linear (Equação 13) converge para a solução exata. Todavia, para rotações moderadas a grandes, a resposta linear diverge da solução exata. 


\section{TRELIÇA DE VON MISES}

A Figura 3-a) apresenta a treliça de von Mises na configuração inicial. Este problema é amplamente empregado pela literatura para validar as formulações que utilizam a não linearidade geométrica. As barras que compõe a treliça tem comportamento elástico linear, conforme ilustrado na Figura 3-c).

Figura 3 - a) treliça de Von Mises na configuração inicial; b) treliça de Von Mises na configuração atual; c) relação constitutiva das barras da treliça.

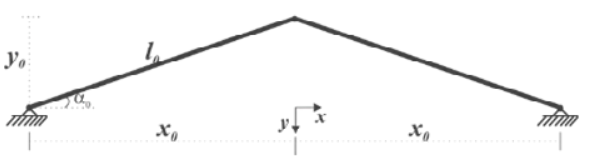

a)

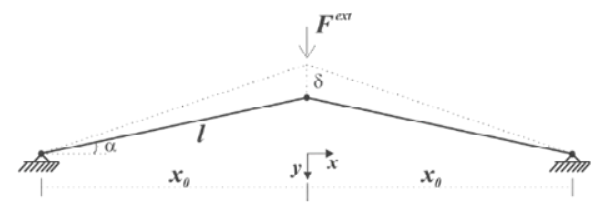

b)

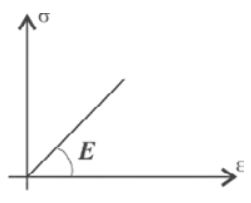

c)

Fonte: Autores.

A energia de deformação é obtida da Equação (12), da qual resulta:

$$
U=2 \cdot\left(\frac{E \cdot \varepsilon^{2}}{2} \cdot A_{0} \cdot l_{0}\right)
$$

Assumindo a hipótese de deformação uniforme ao longo do comprimento da barra, a Equação (11) pode ser escrita como:

$$
\varepsilon=\lambda-1=\frac{l}{l_{0}}-1=\frac{y}{l_{0} \cdot \sin \left[\arctan \left(\frac{y}{x_{0}}\right)\right]}-1
$$

Combinando as Equações (19) e (20), tem-se:

$$
U=A_{0} \cdot l_{0} \cdot E \cdot\left\{\frac{y}{l_{0} \cdot \sin \left[\arctan \left(\frac{y}{x_{0}}\right)\right]}-1\right\}^{2}
$$

A energia mecânica total dessa estrutura fica dada por:

$$
\left.\Pi=P+U=-F^{e x t} \cdot y+A_{0} \cdot l_{0} \cdot E \cdot\left\{\frac{y}{l_{0} \cdot \sin \left[\arctan \left(\frac{y}{x_{0}}\right)\right]}\right\}^{-1}\right\}^{2}
$$


Pelo princípio da estacionariedade, obtém-se a condição de equilíbrio exato:

$$
\frac{\partial \Pi}{\partial \vec{Y}}=-\vec{F}^{e x t}+\vec{F}^{\mathrm{int}}=\overrightarrow{0}
$$

Portanto,

$$
-F^{e x t}+\frac{2 \cdot A_{0} \cdot E \cdot y \cdot\left[-1+\frac{x_{0} \cdot \sqrt{1+\left(\frac{y}{x_{0}}\right)^{2}}}{l_{0}}\right]}{x_{0} \cdot \sqrt{1+\left(\frac{y}{x_{0}}\right)^{2}}}=0
$$

A Figura 4 apresenta a trajetória de equilíbrio desse problema. Assumindo $E=$ $20500 \mathrm{kN} / \mathrm{cm}^{2}, A_{0}=78,5 \mathrm{~cm}^{2}, x_{0}=200 \mathrm{~cm}$ e $y_{0}=10 \mathrm{~cm}$, verifica-se que a carga limite dessa estrutura é igual $77 \mathrm{kN}$. Para esse valor de força externa o determinante da matriz Hessiana é nulo. Caso utilize um controle em força, qualquer acréscimo infinitesimal de carga leva a estrutura a ter um salto de posição de forma abrupta (4 para 21,5 cm). Este fenômeno é denominado pela literatura de snap-thought. Por outro lado, ao usar um controle em posição toda a trajetória de equilíbrio é traçada. Entretanto, a trajetória de equilíbrio entre os deslocamentos de 4 até $21,5 \mathrm{~cm}$ é instável.

Figura 4 - Trajétoria de equilíbrio da treliça de von Mises.

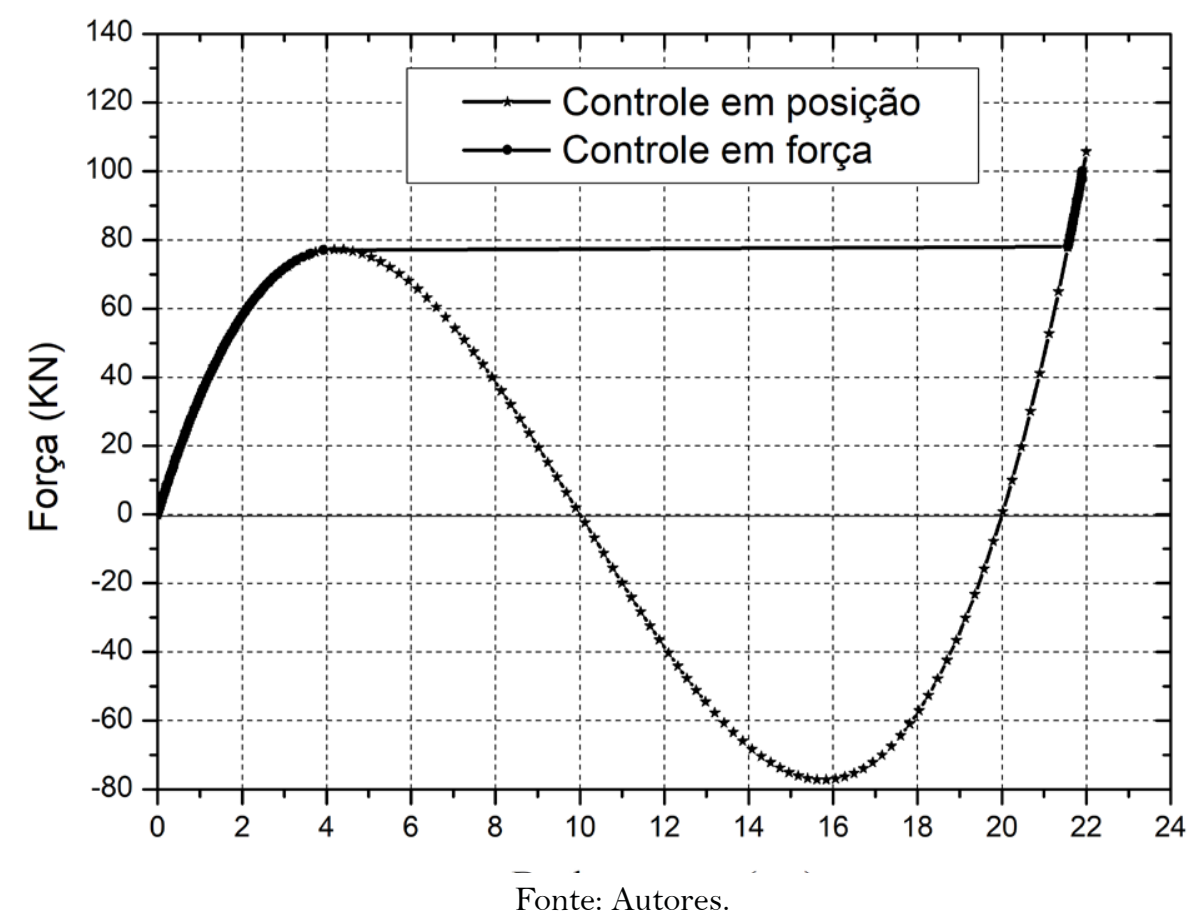




\section{CONSIDERAÇÕES FINAIS}

Este trabalho empregou o método da energia mecânica total com os potenciais escritos em termos das posições nodais para avaliar e debater sobre a trajetória de equilíbrio de elementos de barras simples. Utilizou-se uma descrição Lagrangeana Total para obter a energia de deformação e a medida de deformação longitudinal de engenharia. Observou-se que tanto pelas condições de equilíbrio concretizado na configuração atual, como pela abordagem aqui deduzida, conduziu a trajetória de equilíbrio exato para os problemas analisados. Por fim, em regime de deslocamentos moderados a grandes deve-se realizar uma análise não linear geométrica para mensurar a estabilidade da estrutura com relação aos pontos limites que apareceram na sua trajetória de equilíbrio.

\section{REFERÊNCIAS}

BONET, J.; WOOD, R. D. Nonlinear continuum mechanics for finite element analysis. Cambridge: Cambridge university press, 2008.

CODA, H. B. O método dos elementos finitos posicional: sólidos e estruturas não linearidade geométrica e dinâmica. São Carlos: EESC-USP, 2018.

CRISFIELD, M. A. Non-linear finite element analysis of solids and structures essentials. Chichester, England: John Wiley \& Sons, 1991.

PROENÇA, S. P. B. Análise não linear de estruturas (Notas de Aulas). São Carlos, 2016.

OGDEN, R. W. Non-linear elastic deformations. New York: Dover, 1984. 